\title{
A Region-Growing Algorithm for InSAR Phase Unwrapping
}

\author{
Wei Xu, Member, IEEE, and Ian Cumming, Member, IEEE
}

\begin{abstract}
This paper describes a new region-growing algorithm for interferometric synthetic aperture radar (SAR) phase unwrapping. The algorithm is designed to handle noisy interferograms and based on the following principles.

1) Unwrapping is carried out on the perimeter of "growth regions," and these regions are allowed to grow with consistency checking.

2) Phase information from neighboring pixels is used to predict the correct phase of each new pixel to be unwrapped.

3) Reliability check is applied to each unwrapping attempt, and the threshold used in the check is gradually relaxed so that the most robust unwrapping path is followed.

4) As regions grow into one another, they are merged by adjusting their ambiguity numbers, and further reliability checks must be passed for merging to be allowed.

To verify the proposed algorithm, the region-growing phase unwrapping algorithm has been compared with two of the most widely used algorithms: the cut-line (CL) (also known as residuelinking) algorithm and the weighted least-squares (WLS) algorithm. The FRINGE Sardegna interferogram has been used because it has steep topography that causes problems for many phase unwrapping algorithms. It has been shown that the regiongrowing algorithm can unwrap well into low coherence regions, while making few ambiguity-level errors. This can be attributed to the diversity of growth paths used by the algorithm, in which difficult areas are approached from many directions.
\end{abstract}

Index Terms - Digital elevation model, interferometry, phase unwrapping, region growing, synthetic aperture radar (SAR), terrain mapping.

\section{INTRODUCTION}

$\mathbf{I}$ N SYNTHETIC aperture radar (SAR) interferograms, the phase value of a pixel, after the removal of the component related to the flat earth, is a function of the terrain height and the change in terrain height between SAR passes. However, for variations in the terrain height above a certain minimum, the phase value wraps over multiples of $2 \pi$ and ambiguities occur. Phase unwrapping, the procedure of resolving the ambiguities that are represented by the multiples of $2 \pi$, is then necessary for extracting the information about terrain height, or its change, from the interferogram.

Manuscript received April 8, 1997; revised October 13, 1997. This work was supported by the Natural Sciences and Engineering Research Council, Macdonald Dettwiler and Associates, the British Columbia Advanced Systems Institute, and the British Columbia Research Council.

W. Xu is with MacDonald, Dettwiler and Associates Ltd., Richmond, B.C., Canada V6V $2 \mathrm{~J} 3$.

I. Cumming is with the Department of Electrical and Computer Engineering, University of British Columbia, Vancouver, B.C., Canada V6T 1 Z4 (e-mail: ianc@ee.ubc.ca).

Publisher Item Identifier S 0196-2892(99)00022-4.
Although phase unwrapping has been studied for over 20 years in a number of disciplines, unwrapping of noisy interferograms still presents a challenge [1]. Phase unwrapping of SAR interferograms is particularly difficult because of the sidelooking configuration that causes shadow, foreshortening, and layover. A number of phase unwrapping algorithms for SAR interferograms have been proposed in recent years [2]-[6], however, none of the existing phase unwrapping algorithms are very satisfactory when the phase is noisy or dense fringes occur.

This work introduces a new algorithm based on region growing. ${ }^{1}$ In Section II, the concepts and operation of the algorithm are described. In Section III, an experiment with the Sardegna ERS-1 interferogram is described, in which the performance of the region-growing algorithm is compared with two other representative algorithms. In Section IV, the experimental results are verified with map elevations and further algorithm comparisons are made. The paper finishes with discussions and conclusions in Sections V and VI.

\section{DESCRIPTION OF THE ALGORITHM}

\section{A. Basic Concepts}

In many phase unwrapping algorithms, the accuracy of the result depends on the path chosen to perform the unwrapping. Choosing the best path has been troublesome for many current algorithms. The region-growing algorithm minimizes unwrapping errors by starting at pixels of high data quality and proceeding along dynamic paths where unwrapping confidence is high. Areas that are difficult to unwrap are then approached from a number of directions. Thus, the algorithm is able to correct unwrapping errors to a certain extent and stop their propagation. The algorithm achieves these goals in the following ways.

1) Unwrapping is carried out concurrently in a number of regions. A region is started from a seed where the phase is locally smooth and allowed to grow outwards along controlled data-dependent paths during the unwrapping procedure.

2) Each pixel is unwrapped based on slope predictions made from its unwrapped neighbors. The predictions allow phase changes between two adjacent pixels larger than $\pi$.

\footnotetext{
${ }^{1}$ Some of the concepts of this algorithm have been described in earlier conference papers by the authors [7], [8].
} 


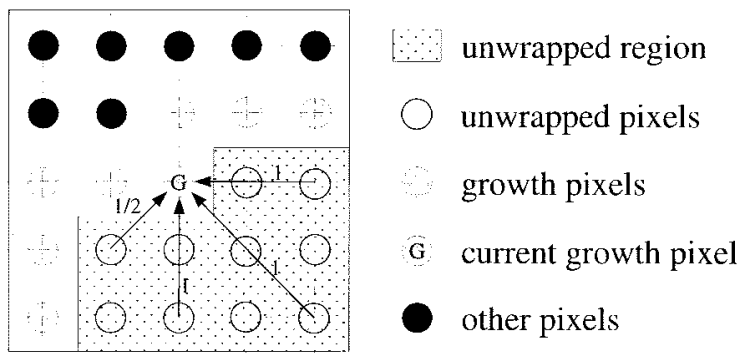

Fig. 1. Growth pixel and its neighbors showing phase prediction directions.

3) Information from as many directions as possible is used to unwrap each pixel. This mitigates the effect of errors in the individual prediction directions.

4) Reliability check based on the consistency of phase predictions is applied to each unwrapping attempt to validate (or temporally disallow) the proposed unwrapping value.

5) Reliability tolerance is gradually relaxed to allow as many pixels as possible to be unwrapped while keeping the unwrapping consistency above a specified level.

6) When regions grow together, an attempt is made to join them by trying many different connecting paths. Consistency checks are applied to ambiguity numbers of the pixels that join the regions. The ambiguity numbers of the merged regions are adjusted accordingly.

The key of the algorithm is to guide the unwrapping along paths that are as reliable as possible. The word reliability or reliable will be used frequently in the paper. Reliability refers to the conditions under which a section of the interferogram will be unwrapped correctly. Reliability is a function of noise and topographic structure of the data. In this algorithm, the areas of least phase noise and the smoothest topography are unwrapped first. The use of multiple growth regions allows difficult areas to be approached from a variety of directions, and allows regions to be merged in a variety of ways. In this way, difficult areas are approached from a solid foundation of correctly unwrapped regions and ambiguity-level errors are minimized. The growth regions can be viewed as islands in the sea of the wrapped interferogram. The depth of the sea is determined by the measures of unwrapping reliability, such as the coherence. As the sea is drained, the islands grow in directions of highest unwrapping reliability and join together. New islands also appear when the sea is drained.

\section{B. Unwrapped Regions and Growth Pixels}

The unwrapping process can be considered a state machine. At each step, the current state consists of one or more contiguous regions of unwrapped pixels. Surrounding each region is a ring of pixels that will be considered for unwrapping in the next iteration of the algorithm. The pixels in this ring will be called growth pixels. An unwrapped region and its ring of growth pixels are illustrated in Fig. 1. At each step of the iteration, one selected growth pixel will be considered for unwrapping, designated with a " $G$ " in Fig. 1. A growth pixel will have one or more unwrapped neighbors, which are used to provide phase predictions, as described in the next section.

\section{How a Growth Pixel is Unwrapped}

Unwrapping of a growth pixel uses phase predictions from a $5 \times 5$ window centered at the growth pixel. Considering the eight neighbors of the current growth pixel, let $N_{u}$ of them be unwrapped.

To provide reliable phase unwrapping, a phase prediction can be made from each of the $N_{u}$ unwrapped neighbors. $N_{u}$ is generally required to be larger than one, except near a seed. The predictions are made along lines, as illustrated in Fig. 1, where $N_{u}=4$. The prediction $\phi_{k}^{p}$ is formed from the $k$ th unwrapped neighbor, and it is either a linear or constant prediction, depending on the number of unwrapped pixels along the prediction line, as follows.

1) If two unwrapped pixels are available along the prediction line, a linear prediction is used

$$
\phi_{k}^{p}=2 \phi[k]-\phi\left[k^{\prime}\right]
$$

where $k^{\prime}$ stands for the next unwrapped pixel along the prediction line.

2) Otherwise, the prediction is simply given by the phase value at pixel $k$

$$
\phi_{k}^{p}=\phi[k] .
$$

Then, a composite prediction $\phi^{p}$ is formed as a weighted average of the $N_{u}$ individual predictions

$$
\phi^{p}=\left(\sum_{k=1}^{N_{u}} w_{k} \phi_{k}^{p}\right) /\left(\sum_{k=1}^{N_{u}} w_{k}\right)
$$

where $w_{k}$ is one for case 1 because it is more reliable and 0.5 for case 2. The weights are shown as the numbers beside the corresponding prediction line in Fig. 1.

The phase prediction (3) is used to attempt to unwrap the growth pixel. The proposed unwrapped phase value $\phi_{u}$ at the growth pixel is computed as

$$
\phi_{u}=\phi_{w}+2 \pi m
$$

where $\phi_{w}$ is the wrapped phase at the growth pixel and $m$, the ambiguity number, is

$$
m=n i n t\left(\frac{\phi^{p}-\phi_{w}}{2 \pi}\right)
$$

where $\operatorname{nint}(x)$ is the integer closest to $x$. The result of the unwrapping attempt is accepted or rejected based on a measure of reliability discussed below.

As the unwrapping is based on the composite prediction rather than any of its neighbors alone, a phase change larger than $\pi$ between two adjacent pixels is possible. In this way, it is possible for the algorithm to correctly resolve aliased phase values, which can occur in areas of steep topography.

A numerical example is shown in Fig. 2. The original wrapped phase values in a $5 \times 5$ window centered on the growth pixel are shown in Fig. 2(a), where the range of phase values $[0,2 \pi)$ is quantized to $[0,100)$ for easy representation and the center pixel in parentheses represents the growth pixel. Suppose the corresponding unwrapped phase values are shown in Fig. 2(b), where " $x$ " represents a pixel that has not yet been 


\begin{tabular}{|c|c|c|c|c|}
\hline 85 & 15 & 25 & 30 & 75 \\
\hline 75 & 10 & 25 & 50 & 90 \\
\hline 60 & 5 & $(20)$ & 75 & 20 \\
\hline 45 & 90 & 0 & 35 & 80 \\
\hline 40 & 70 & 90 & 20 & 50 \\
\hline
\end{tabular}

(a)

\begin{tabular}{|c|c|c|}
\hline$x$ & $x$ & $x$ \\
\hline$x$ & $G$ & 130 \\
\hline 90 & 110 & 120 \\
\hline
\end{tabular}

(c)

\begin{tabular}{|c|c|c|c|c|}
\hline$x$ & $x$ & $x$ & $x$ & $x$ \\
\hline$x$ & $x$ & $x$ & $x$ & $x$ \\
\hline$x$ & $x$ & $G$ & 175 & 220 \\
\hline$x$ & 90 & 100 & 135 & 180 \\
\hline$x$ & 70 & 90 & 120 & 150 \\
\hline
\end{tabular}

(b)

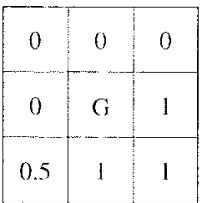

(d)
Fig. 2. Numerical example: (a) wrapped phase value of neighbors, (b) unwrapped phase value of neighbors, (c) individual prediction from neighbors, and (d) individual prediction weights.

unwrapped and "G" represents the current growth pixel. Then the corresponding predictions along the four directions are given in Fig. 2(c), and the corresponding prediction weights are given in Fig. 2(d). The composite prediction is then

$$
\phi^{p}=\frac{0.5 \times 90+110+120+130}{0.5+1+1+1} \approx 115.7
$$

and the ambiguity number is

$$
m=\operatorname{nint}\left(\frac{115.7-20}{100}\right)=1 \text {. }
$$

Thus, the result of this unwrapping attempt is

$$
\phi_{u}=20+100 \times 1=120 .
$$

In this example, the phase difference between the current growth pixel and its neighbor on the right side is larger than $\pi$, and this algorithm successfully unwrapped the phase by using phase predictions along multiple directions. The application of phase predictions allows the algorithm to accept a phase difference between neighboring pixels of more than $\pi$ in its normal unwrapping procedure.

Note that this unwrapping procedure changes only the ambiguity number, not the fractional phase value of the pixel.

\section{Measures of Unwrapping Reliability}

The attempt at unwrapping the growth pixel is accepted only when a reliability test is passed. There are many possible measures of reliability. Three of them that have been used in our experiments are as follows.

Measure 1: It uses the average deviation $d_{p}$ of the individual predictions

$$
d_{p}=\left(\sum_{k=1}^{N_{u}} w_{k}\left|\phi_{k}^{p}-\phi^{p}\right|\right) /\left(\sum_{k=1}^{N_{u}} w_{k}\right) .
$$

The reliability test is passed when

$$
d_{p}<t_{p}
$$

The threshold $t_{p}$ may be gradually relaxed from a small value (e.g., $\pi / 4$ ) to a larger value $\leq 2 \pi$ (e.g., $\pi / 2$ ) in successive iterations so that reliable data will pass the test early and thus be unwrapped earlier.

Measure 2: It uses the difference $d_{u}$ between the unwrapping result $\phi_{u}$ and the composite prediction $\phi^{p}$

$$
d_{u}=\left|\phi_{u}-\phi^{p}\right|
$$

The reliability test is passed when

$$
d_{u}<t_{u}
$$

The threshold $t_{u}$ may be gradually relaxed from a small value (e.g., $\pi / 4$ ) to a larger value $\leq \pi$ (e.g., $\pi / 2$ ) in successive iterations.

Measure 3: It uses the local coherence $c$ of the interferogram, ${ }^{2}$ also called the correlation coefficient [9]

$$
c=\left|E\left\{a_{m} e^{j \phi_{m}} a_{s} e^{-j \phi_{s}}\right\}\right| / \sqrt{E\left\{a_{m}^{2}\right\} E\left\{a_{s}^{2}\right\}}
$$

where $E\{x\}$ is the average value of $x, a_{m}$, and $a_{s}$ are the amplitudes, and $\phi_{m}$ and $\phi_{s}$ are the phases of the master and slave SLC images, respectively. The average is typically calculated in a window with an area of 16 pixels or more. If the amplitude values are not available when coherence is calculated, an approximation can be obtained by assuming constant amplitude, which leads to

$$
c \approx\left|E\left\{e^{j \phi_{m}} e^{-j \phi_{s}}\right\}\right|=\left|E\left\{e^{j \phi_{w}}\right\}\right| .
$$

The reliability test is passed when

$$
c>t_{c} \text {. }
$$

The threshold $t_{c}$ may be gradually relaxed from a large value $\leq 1$ to a small value $\geq 0$.

Several ways have been tried to combine these measures of reliability. Experiments have shown that the following two combinations work well.

Test 1: The applications of measures 1 and 2 are combined by simply having $t_{u}=t_{p}$ so that the two thresholds can be relaxed simultaneously, and both of the measures must pass their tests for the unwrapping result to be accepted.

Test 2: The first two thresholds are kept at a common constant

$$
t_{u}=t_{p}=\text { constant }
$$

while $t_{c}$ is a variable and all of the three measures must pass their respective tests. A typical value of this constant is $\pi / 2$.

For the above numerical example, we have (17) and (18), shown at the bottom of the next page. It has been found that phase unwrapping is quite reliable when the threshold of $t_{p}$

\footnotetext{
${ }^{2}$ The coherence is a measure of the local phase noise in the interferogram, so high coherence is an indicator of phase prediction reliability.
} 
and $t_{u}$ are between $\pi / 4$ and $\pi / 2$. Thus, the unwrapping is quite reliable in the example above.

\section{E. Process of Region Growing}

Phase unwrapping is carried out concurrently but independently in a certain number of regions (e.g., 255). An unwrapping record is kept for each pixel. The unwrapping record indicates in which regions the pixel has been unwrapped (at most two for the current program) and the corresponding ambiguity number for each of the regions.

Each region begins from a single pixel, one of a set of selected seeds. The seeds are distributed throughout the interferogram based on a suitability criterion, such as local coherence. An arbitrary ambiguity number (e.g., 128) is assigned to each seed. Regions and their seeds are ordered so that those with high reliability are assigned a small index number. The reliability of a region may be measured by the coherence of its seed, the size of the region, or the averaged measures of unwrapping reliability, as described in Section II-D. The coherence at its seed has been used in our experiment for simplicity. An example of the distribution of ten seeds and the regions grown from the seeds is shown in Fig. 3(a).

The regions are allowed to grow within an iterative algorithm structure. There are two levels of iteration in the algorithm. The outer iteration is called the threshold relaxing iteration, and the inner iteration is called the growth iteration. In each growth iteration, growth rings are defined for the unwrapped regions and the algorithm tries to unwrap each growth pixel in the growth rings once. Newly unwrapped rings are established after each growth iteration, as shown in Fig. 3(b). The gaps in the newly unwrapped rings are pixels that have failed the reliability test. The regions before growing are dotted, and the overlaps that occur in the newly unwrapped rings are shaded in Fig. 3(b). Then, new growth rings are created and unwrapping is tried again using the same thresholds. When a growth iteration fails to unwrap any new pixels, the growth iteration is terminated. Then the thresholds are relaxed by one step, and a new threshold relaxing iteration begins. In this way, the pixels with highest reliability are unwrapped first. Thus, the unwrapping path is data dependent and as reliable as possible.

\section{F. Region Merging}

As each of the regions grows individually, two or more regions may develop an overlap area. Once such overlap areas grow to a minimum size (e.g., three pixels), attempts to merge the overlapped regions are made.

Suppose regions $i$ and $j$ have an overlap area of $N_{o v}$ pixels. The difference between the ambiguity number $m_{i}$ for region $i$ and the ambiguity number $m_{j}$ for region $j$, originating from

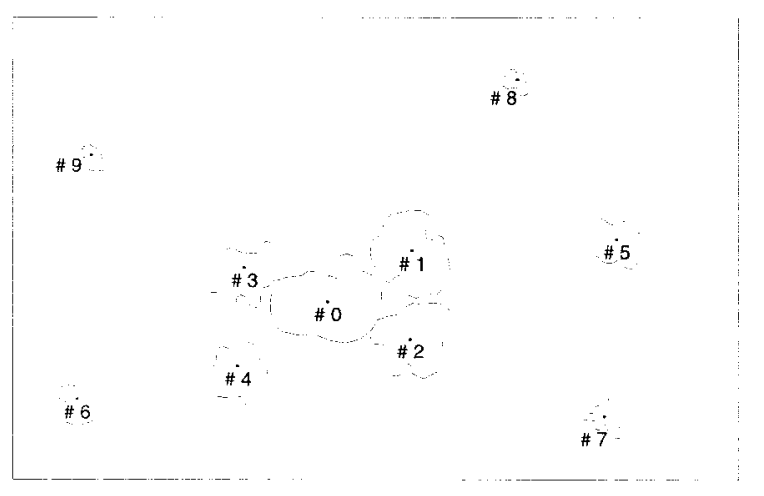

(a)

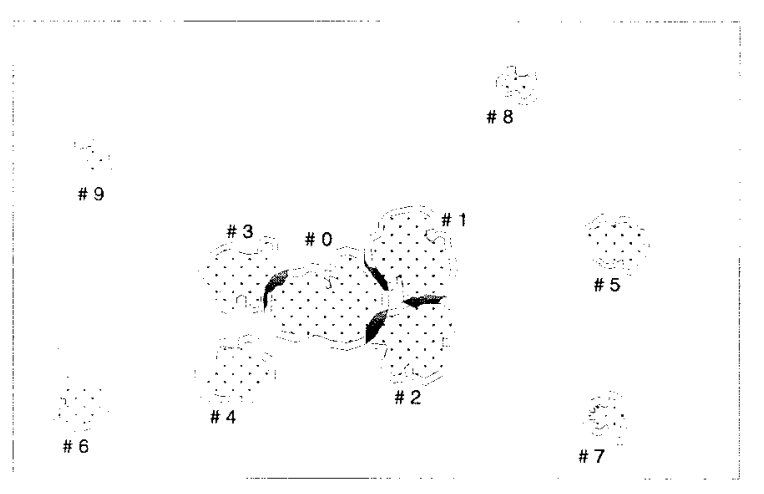

(b)

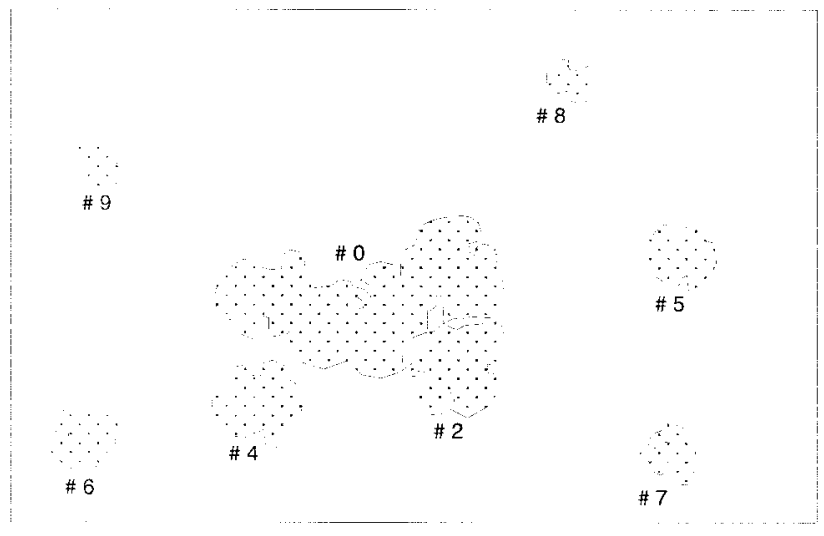

(c)

Fig. 3. Examples of (a) regions grown from seeds, (b) newly unwrapped rings with overlaps, and (c) merged regions and cleared overlaps.

a different seed, is examined at each pixel in the overlap area. Their mode $D_{m d}$ is then a reasonable estimate of the difference between the ambiguity number for the two regions.

The reliability of this estimate is then checked by the following procedure. The difference between the ambiguity number for the two regions at each pixel is compared with $D_{m d}$. Suppose there are $N_{c}$ pixels in the overlap area that

$$
\begin{aligned}
d_{p} & \approx \frac{0.5 \times|90-115.7|+|110-115.7|+|120-115.7|+|130-115.7|}{0.5+1+1+1} \times \frac{2 \pi}{100} \\
& \approx 0.2 \pi \\
d_{u} & \approx|120-115.7| \times \frac{2 \pi}{100}=0.086 \pi
\end{aligned}
$$


agree with $D_{m d}$. The estimation of the difference between the ambiguity number for the two regions $D_{m d}$ is considered to be reliable as long as the following two threshold tests are passed:

$$
N_{c} \geq t_{r n}
$$

and

$$
N_{c} / N_{o v} \geq t_{r r}
$$

An example of the value of the threshold $t_{r n}$ is three and of the threshold $t_{r r}$ is $3 / 4$.

If the estimation of the difference between the ambiguity number for the two regions is reliable, the two regions are then merged by moving the pixels in the region with the larger index to the region with the smaller index. The pixels in the overlap area, which do not agree with the estimate of the difference between the ambiguity numbers, are cleared by resetting the corresponding unwrapping record. The large index is freed after the merging. If the estimation of the difference between the ambiguity numbers for the two regions is not considered reliable, the two regions are kept separate and the whole overlap area is cleared by resetting the corresponding unwrapping record.

An example of merged regions and cleared overlaps is shown in Fig. 3(c). Regions \#1 and \#3 have been merged with region $\# 0$, but some of the pixels in the overlap areas may have been cleared if they do not agree with the majority. In contrast, region \#2 is kept separate due to a failure in threshold tests, and the corresponding overlap areas are cleared.

One advantage of this algorithm is that it allows region $\# 2$ to be merged into region $\# 0$ at a later stage if the reliability measure improves. In subsequent growth iterations, a larger overlap area will hopefully be generated, allowing the reliability measure to increase.

Whenever vacancies of region indexes appear after region merging, additional regions can be started from newly selected seeds. The existing region indexes are compressed to lower values, and the new seeds are assigned higher indexes, as they are considered less reliable.

\section{UNWRAPPING THE SARDEGNA INTERFEROGRAM}

An experiment using the region-growing algorithm to unwrap the difficult Sardegna ERS-1 SAR interferogram is described in this section. Many researchers in the FRINGE group have worked with it, but very few successful results have been reported. The only results published are two unwrapped images presented in the ERS-1 FRINGE workshop without verification [10]. The main reason for this lack of success is that the interferogram is difficult for phase unwrapping algorithms like the commonly-used "residue-linking" or "cutline" (CL) algorithm [2], [4], [11]. Unwrapping with two other algorithms are also given for comparison. The algorithms were chosen as they were the most popular ones when this paper was written. Since then, many other promising algorithms have been proposed [12]-[17].

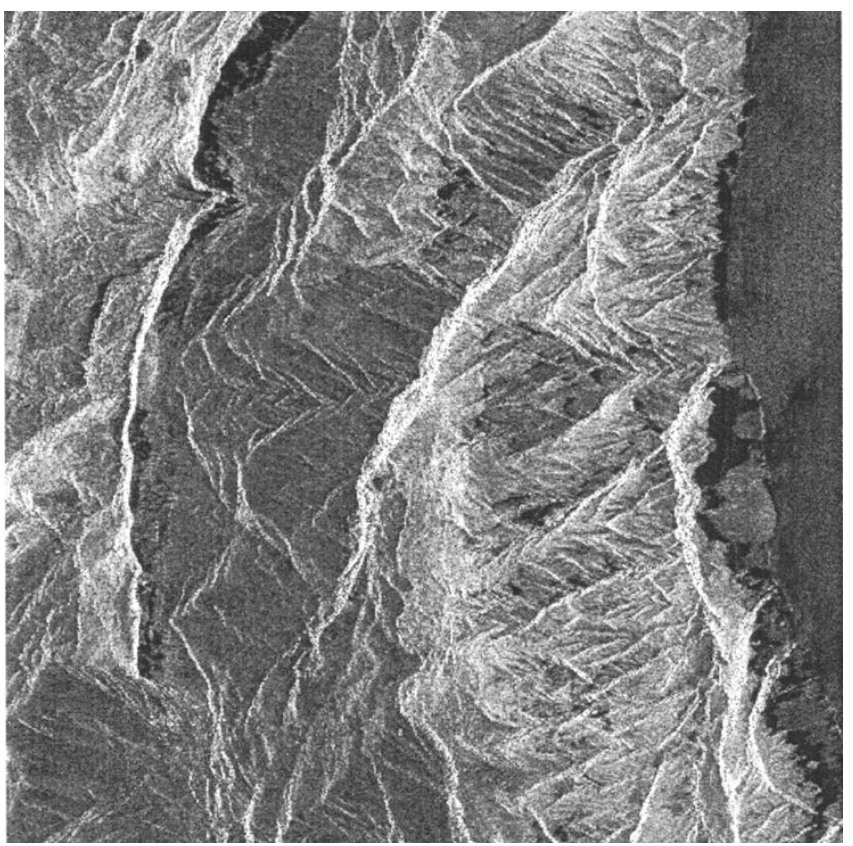

Fig. 4. Magnitude of the Sardegna ERS-1 interferogram.

\section{A. Sardegna ERS-1 Interferogram}

ERS-1 data of Sardegna, Italy, collected on August 2, 1991 (orbit 241), and August 8, 1991 (orbit 327), was used. Attention is directed to a portion of frame 801 with dimensions of 8 and $16 \mathrm{~km}$ in slant range and azimuth, respectively. It is toward the far range of the full scene, centered at $40^{\circ}$ $8^{\prime} \mathrm{N}, 9^{\circ} 32^{\prime} \mathrm{E}$. This $512 \times 512$ pixel magnitude image is shown in Fig. 4, after smoothing by $2 \times$ in range and $8 \times$ in azimuth. The averaged pixel size is $16 \times 32 \mathrm{~m}$, giving approximately square pixels on the ground. Range increases from left to right. The main part of the scene is quite mountainous, with the Mediterranean Sea along the right edge of the scene. According to the FRINGE baseline listings [18], the perpendicular baseline is $-126 \mathrm{~m}$.

The SAR signal data were processed to SLC images, registered, approximately flattened, and the interferogram was formed. The phase of the interferogram is shown in Fig. 5, with the corresponding local coherence shown in Fig. 6. Phase smoothing and the coherence calculation are based on the 2:1 and 8:1 range and azimuth averaging ratios. Such an averaging ratio should not be too large that much phase discontinuity information is lost nor too small that the coherence estimation is too inaccurate. Although the averaging ratio used was lower than optimal for accurate estimation of coherence, it was enough to be used in this unwrapping example, as there are other reliability tests applied as well.

This SAR interferogram is considered to be difficult to unwrap because of its large areas of low coherence, which are mainly caused by layover due to the extreme local topography. These areas of low coherence segment the interferogram into many pieces, which creates structural difficulties for the unwrapping algorithms.

The distribution of the residues is shown in Fig. 7. The 440 columns on the left side of the interferogram are in the 


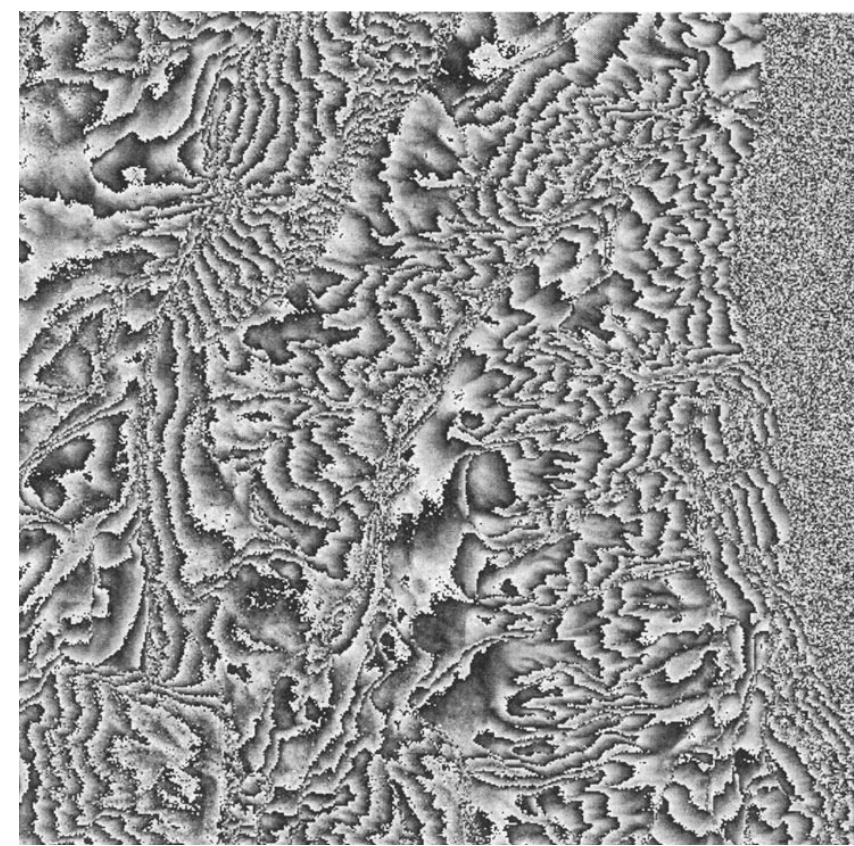

Fig. 5. Phase of the Sardegna ERS-1 interferogram before unwrapping.

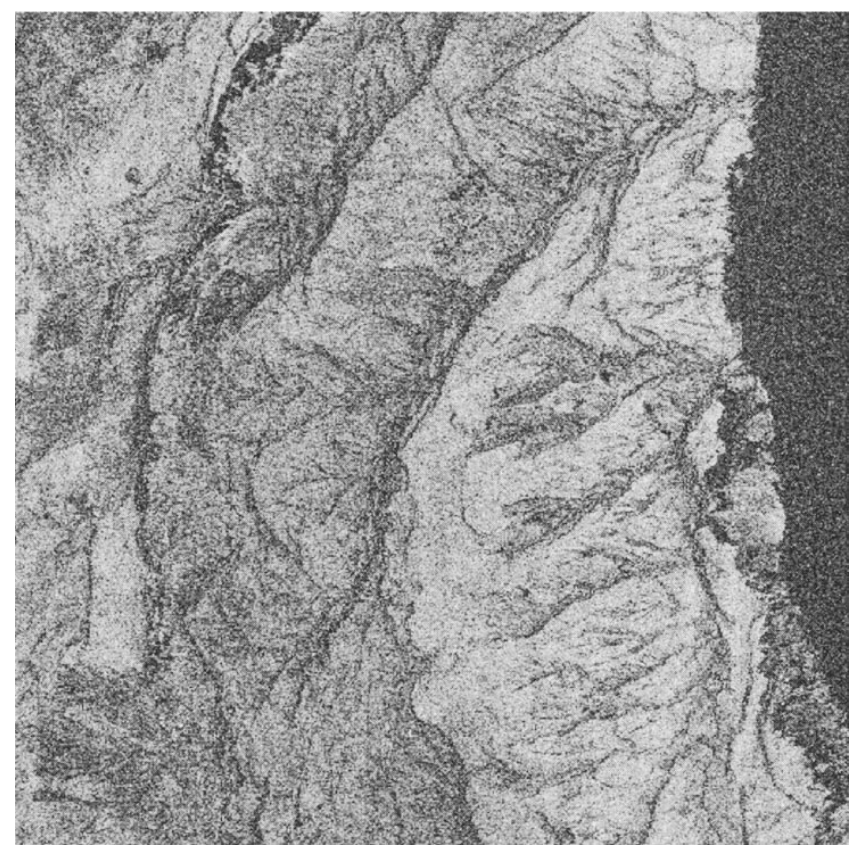

Fig. 6. Coherence of the Sardegna ERS-1 interferogram.

land area (excluding the Mediterranean sea). There are 9676 residues or phase singularities found in this land area, which correspond to a residue density of $4.31 \%$. This interferogram is difficult to unwrap with "residue-linking" algorithms [2], [4]. In these algorithms, pairs of "residues" must be correctly linked, and this is difficult with such a high-residue density [11]. The "edge detection" algorithm [3] runs into difficulty as well, when edges join together or when edges are disjoint. The "least-squares" algorithms [5] will likely underestimate the phase slope where large global phase discontinuities exist.

The Sardegna interferogram was unwrapped using the region-growing algorithm described above. For comparison,

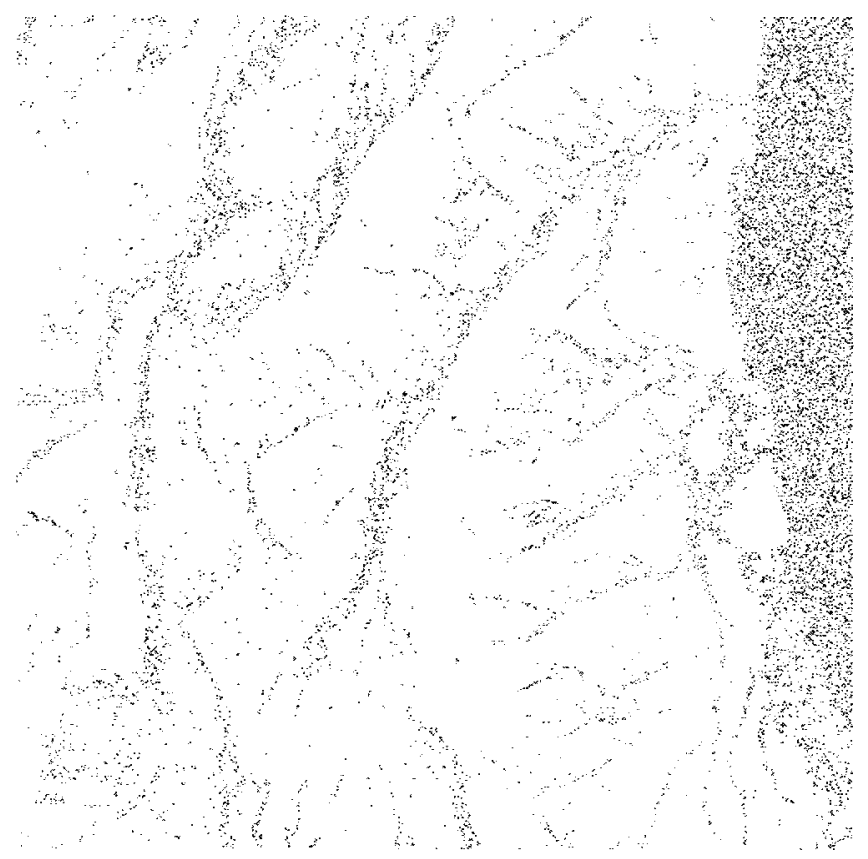

Fig. 7. Distribution of "residues" in the Sardegna ERS-1 interferogram.

it is also unwrapped using two other unwrapping algorithms, which are in popular use nowadays.

\section{B. Unwrapping with the Region-Growing Algorithm}

A total of 255 initial seeds were selected based on regional diversity and highest coherence. Growth pixels were checked for unwrapping reliability using the following thresholds:

1) constant average deviation threshold $t_{p}=\pi / 2$;

2) constant prediction difference threshold $t_{u}=\pi / 2$;

3) variable local coherence threshold $t_{c}$, which starts at $200 / 256$ and is gradually relaxed to zero.

These reliability tests for pixel unwrapping are combined using Test 2 described in Section II-D. Unwrapped regions are joined using the following reliability thresholds:

1) number of pixels agreeing with the ambiguity number, $t_{r n}=3$

2) fraction of pixels agreeing with the ambiguity number, $t_{r r}=3 / 4$.

The unwrapped phase is shown in Fig. 8. The displayed result is the region with index 0 , and its brightness is scaled by a factor of $1 / 30$ because the unwrapped phase spans 30 ambiguity intervals. The total number of pixels in this region with index 0 is 218373 .

The following statistics are obtained for the land area. The total number of unwrapped pixels in this region is 206019 , corresponding to about $91.45 \%$ of this land area. The total number of pixels in all unwrapped regions is 209352, corresponding to about $92.93 \%$ of this land area.

Note that the percentage of unwrapped pixels can be increased to $100 \%$ and the merging of all regions can be completed by lowering the associated reliability thresholds. However, the quality of the unwrapped product will suffer. In any case, the choice of the threshold can be fine tuned to suit the user's objectives. 


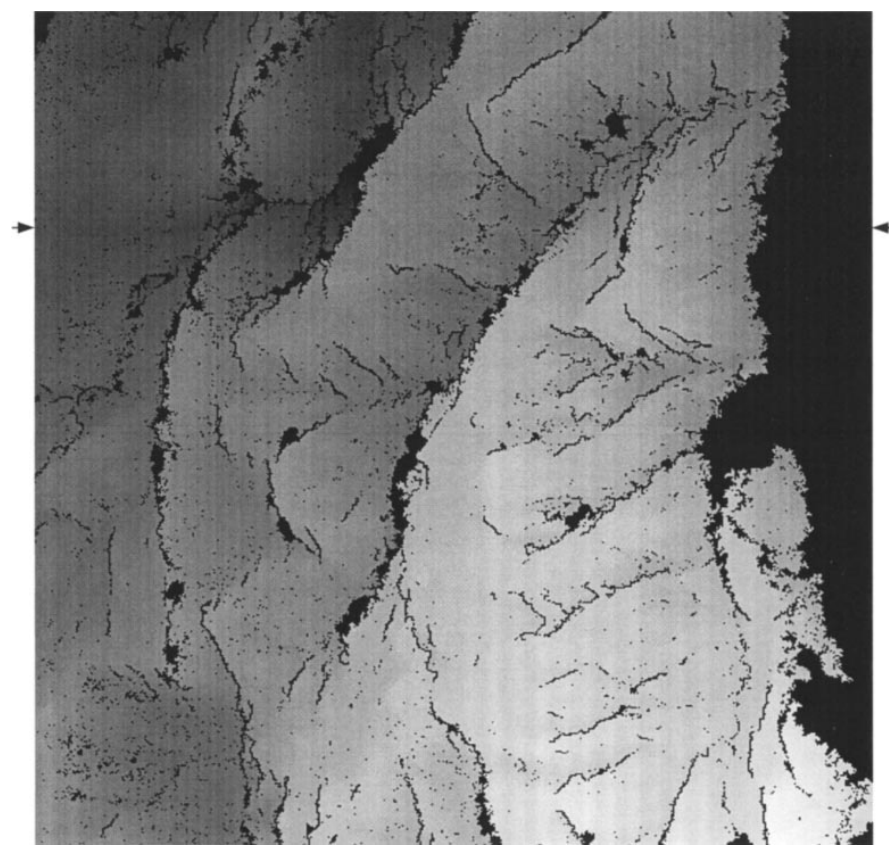

Fig. 8. Unwrapped phase of the Sardegna ERS-1 interferogram using the region growing algorithm, region $\# 0$.

While the iteration strategy and the computer coding have not optimized, the algorithm does require more computing time than other phase unwrapping algorithms. This is because of the detailed attempts to try different unwrapping paths and the associated reliability tests that are carried out.

All of the pixels that could not be unwrapped, and pixels that belong to other regions, are shown in black in Fig. 8. The pixels that could not be unwrapped are located in areas of low SNR, temporal correlation, or layover, so that none of the unwrapped regions could reach them in the growth iterations. Regions with an index greater than zero were isolated in noisy areas so that they could not be merged with region $\# 0$.

The algorithm automatically recognizes that the lowcoherence sea area on the right-hand side should not be unwrapped. The low-coherence regions caused by layover in the land area are prominent in the figure, as they are also left wrapped, although small isolated unwrapped areas may be present in some of the black areas.

Our present implementation of the region-growing algorithm devotes a lot of time to generating phase predictions and doing reliability checking. This improves algorithm robustness at the expense of computing time. This makes our present implementation much slower than the CL algorithm. However, Pritt has implemented a version of the region-growing algorithm with computing times comparable with the other algorithms [17].

\section{Comparison with the CL and the Weighted Least-Squares (WLS) Algorithms}

The CL algorithm has a number of variations and implementations. New variations and implementations are continuing to emerge [15], [17]. Only the best implementation available to us when the research was performed was used in this paper.
Unwrapping by the CL algorithm was performed by Joughin at JPL [19], using a modified version of the original CL algorithm [2]. The phase of the largest unwrapped region is shown in Fig. 9.

Several black linear features are noted in Fig. 9, for example, the three-part line segment around range cell 385, range line 362 (counting from the top left of the image). These lines occur when CL's connect residues that are fairly far apart. They represent unwrapping discontinuities across which ambiguity errors may occur.

The difference between the unwrapped data in Figs. 8 and 9 is shown in Fig. 10. Differences within the range $(-\pi, \pi)$ are shown with a linear grayscale, and those out of this range are shown white. Black indicates that data are not available. This area is chosen as the portion of interest because it is in the middle of the area studied in the next section.

In the central part of the interferogram, where the coherence is generally high and the topography is not too steep, the two algorithms agree generally, except a difference of one ambiguity interval $(2 \pi)$ between the upper and lower parts. However, other areas, like the areas at the top left of the interferogram, which are almost completely isolated from the central part by ridges of steep topography, have a large ambiguity difference.

Unwrapping by the WLS algorithm was performed at DLR [20], and the resulting unwrapped phase is shown in Fig. 11. The WLS algorithm gives a result that is pleasing in appearance because of its completeness and smoothness, but it is clear that misleading results are obtained in the sea area and in places of sharp topography.

The difference between the valid data in Figs. 8 and 11 is shown in Fig. 12. In the central part of the interferogram where the average coherence is high and the discontinuities are not too large, the two algorithms generally agree up to the one ambiguity level, as differences of fractions of an ambiguity are common due to the smoothing operation of the WLS algorithm. However, in the left part of the interferogram, which is almost completely isolated from the central part by significant topographical features, the disagreement between the two algorithms is larger than one ambiguity interval.

\section{VERIFICATION WITH MAP ELEVATIONS}

In the central part of the interferogram where coherence is generally high and the topography is not too extreme, the three algorithms give comparable results. However, in the left part of the interferogram, which is almost completely isolated from the central part by a ridge of steep terrain, the disagreement between the three algorithms is obvious. In these cases, ground truth can be used to understand and resolve the differences, thereby helping to evaluate the unwrapping quality of the three algorithms.

As digital elevation model (DEM) data for the whole interferogram were not available, elevation data from a $1: 25000$ topographic map for one range line is used to compare the algorithms. Range line 127 (see the arrows in Fig. 8 for the position) is chosen because all three algorithms unwrapped 


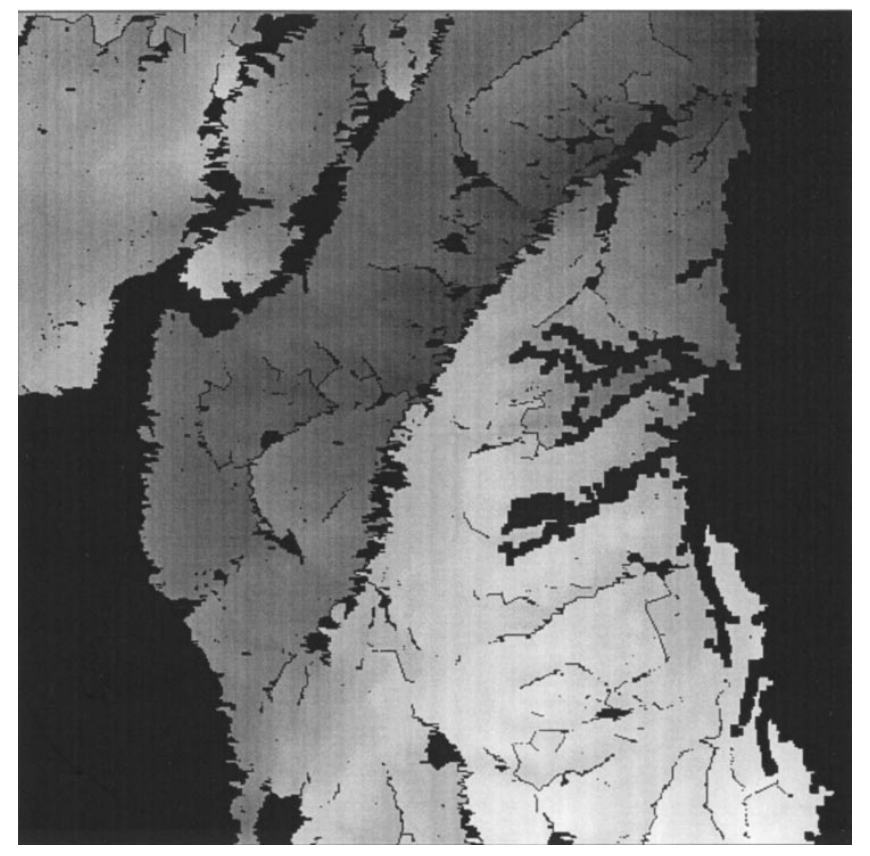

Fig. 9. Unwrapped phase of the Sardegna ERS-1 interferogram using the CL algorithm.

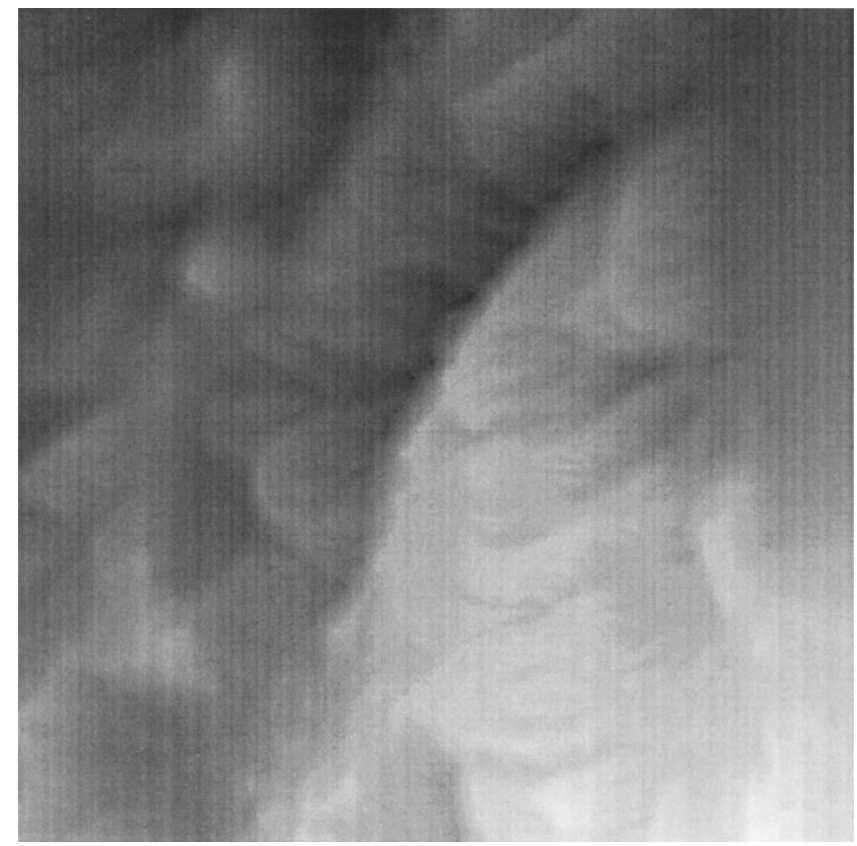

Fig. 11. Unwrapped phase of the Sardegna ERS-1 interferogram using the WLS algorithm.

most of the pixels on this line and challenging significant topographic features are present along the line.

\section{A. Comparison of Phase Values}

As the interferogram is not accurately flattened, it is useful to begin by comparing the unwrapped phase of the three algorithms along the selected range line. This is a fair comparison because the imperfect flattening affects all phase images equally. The comparison of phase along the range line is shown in Fig. 13. Gaps in the RG and CL curves indicate where

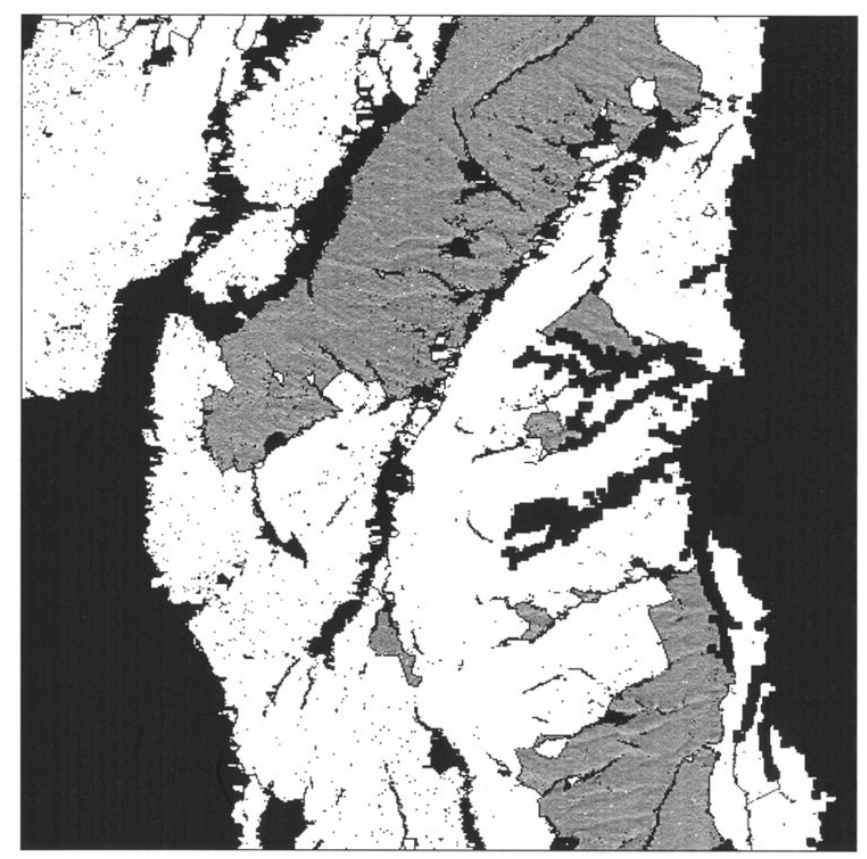

Fig. 10. Difference between the unwrapped phase of the region-growing algorithm and cut line algorithm with a fit to the upper middle portion.

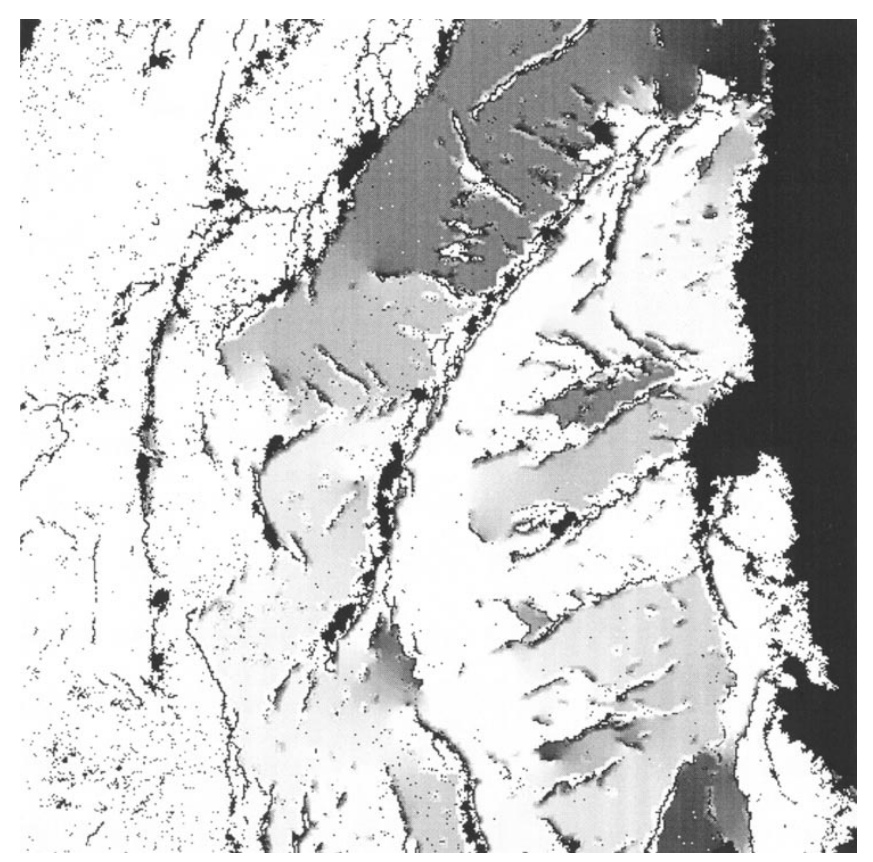

Fig. 12. Difference between the unwrapped phase of the region-growing algorithm and the WLS algorithm.

the phase values are not unwrapped (or are unwrapped into unjoined regions). The following observations can be made for each group of range cells (RC).

1) RC 196-310: The best agreement occurs in this area because it is here that the datum was chosen to align the ambiguity levels of the three algorithms. The WLS exhibits smoothing in the lower-coherence area around RC 275.

2) RC 1-105 and RC 131-165: The largest differences are evident in this area, differences of 11 ambiguities 


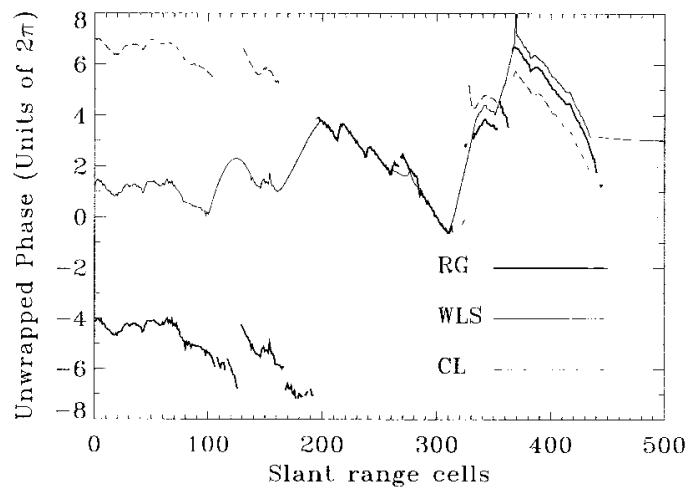

Fig. 13. Unwrapped phase of range line 127.

between the CL algorithm and the region-growing algorithm, and 5.5 or 6.5 ambiguities between the WLS algorithm and the region-growing algorithm.

3) RC 106-130 and RC 166-195: The region-growing algorithm worked through or around the low-coherence areas, but the other algorithms did not. The WLS algorithm smoothed over these areas, while the $\mathrm{CL}$ algorithm left it wrapped.

4) RC 326-440: The three algorithms are roughly one ambiguity apart in this area, with the CL algorithm losing two ambiguities relative to the other two algorithms at the break near RC 365 .

5) RC 440-512: This is the area of the sea, which only the WLS attempts to unwrap, yielding a smooth result.

Note that the WLS algorithm provides a smooth but often inaccurate result over regions of low coherence. See, for example, the region between range cells 106 and 130 where the slope has been reversed with respect to the region-growing algorithm. This effect is not unlike the effect observed by Bamler et al. [21], where they observed "cycle slips" in the WLS algorithm in regions of low coherence and high surface slope.

In Fig. 13, the fine structure of the phase of the three results agree reasonably well, but major differences exist in the ambiguity levels. The map elevations will be used to identify which of these solutions is best.

\section{B. Comparison with Elevations from the Map}

Before a comparison can be made with the map elevations, the flattening of the phase values must be improved. This was arbitrarily done by adding the same constant slope to the unwrapped phase of each result so that the phase in the high-coherence regions of RC 196-310 fit the map elevations closely.

After this flattening refinement and scaling the phase to elevation, the phase-derived elevations are compared with the map elevations in Fig. 14. One ambiguity of $2 \pi$ corresponds to about $70 \mathrm{~m}$ of elevation change.

Note that over most of the figure, the phase-derived elevations are parallel to the map elevation. This attests to the effectiveness of the flattening operation. Note also that the slant range axis sometimes reverses itself in the case of the map data. This is a result of the ground range to slant range

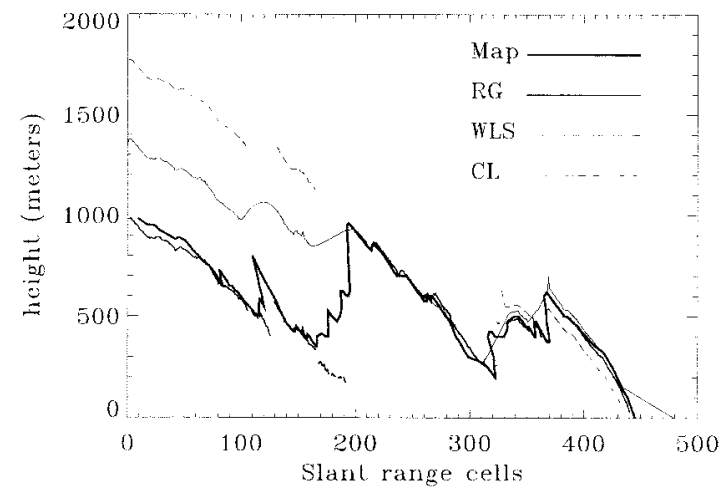

Fig. 14. Comparison of InSAR-derived heights with map elevations.

conversion used when interpreting the map data and indicates regions of SAR data layover.

From the comparison in Fig. 14, the following observations can be made.

1) RC 196-310: All algorithms agree closely with the map in the left half of this high-coherence region. In the right half, small differences with the map are observed, which could be due to errors in reading the map or due to the radar scattering properties.

2) RC 1-105 and RC 130-165: The region-growing algorithm agree closely with the map in these regions because it has been most successful in propagating the absolute phase from the central region. The more limited abilities of the WLS and CL algorithms to follow absolute phase over difficult regions has resulted in offsets of about 5.5/6.5 and 11 ambiguities (about 400 and $800 \mathrm{~m}$ ), respectively.

3) RC 106-130 and RC 166-195: The region-growing algorithm is the only one to effectively unwrap into the gaps. It agrees closely with the map in RC 106-115, but it makes up to five ambiguities (about $350 \mathrm{~m}$ ) local mistakes in the other areas in doing so.

4) RC 326-440: The region-growing algorithm and WLS algorithms both work well in this region, with WLS giving a slightly better result. The $\mathrm{CL}$ algorithm is more bothered by the steep topography near RC 365 , losing two ambiguities (about $150 \mathrm{~m}$ ).

\section{DISCUSSION}

\section{A. Map Comparison}

In this comparison with map data, the region-growing algorithm has been proven to be less susceptible to large errors that can occur in noisy areas of the interferogram. This is largely because of the ability of the region-growing algorithm to venture deeper into noisy regions, attempting to work its way through from many different directions. As a result, the comparison illustrates the effectiveness of the region-growing algorithm in unwrapping difficult areas.

In our opinion, this implementation of the $\mathrm{CL}$ algorithm results in unwrapping errors because it cannot avoid linking the wrong pairs of residues when the residue density is high. Also, the WLS algorithm results in unwrapping errors because 
it tries to fit the unwrapped phases to a smooth model in lowcoherence areas, which is not valid in areas of significant topographic changes.

While these detailed observations were made after examining one line of map data, it can be inferred from the unwrapping structure seen in Figs. 8-12 that our observations will likely apply to other areas of the interferogram. Four other range lines were examined with very similar results [22].

\section{B. Algorithm Operation}

Note that the growth pixel reliability tests are combined in two different ways. In the combination test described in Section II-D, only the deviations of the predictions and the difference between the prediction and the unwrapped value are used and they are relaxed simultaneously. In the combination Test 2, the deviation and prediction thresholds are kept constant and the local coherence is added to the test and relaxed gradually. It was found that Test 1 allows more pixels to be unwrapped, while Test 2 results in a little more fidelity in the unwrapped regions. It may be better to apply Test 2 first and then apply Test 1 after the minimum tolerance in Test 2 has been reached.

Phase changes larger than $\pi$ between two adjacent pixels are not strictly prohibited in this algorithm. This feature of the algorithm allows it to deal with areas with steep topography where dense fringes occur.

\section{CONCLUSIONS}

A new phase unwrapping algorithm has been developed. The new algorithm uses phase predictions and consistency checks to guide the unwrapping path along directions where the unwrapping is most reliable. The unwrapping paths support the growth of contiguous regions, which are eventually merged with other regions to complete the unwrapping process. Unwrapping errors in difficult areas are thus minimized by approaching each area from a number of directions. Regions are joined reliably by trying many different connecting paths.

The region-growing phase unwrapping algorithm has been compared with two of the most popular algorithms. The ERS1 Sardegna image has been used for the comparison because it has extreme topography, which has caused problems for many phase unwrapping algorithms. It has been shown that for interferograms with extreme local topography, like the Sardegna interferogram, the region-growing algorithm can successfully unwrap further into the low-coherence regions than either of the implementations of CL and WLS algorithms that we have used for our comparison and makes fewer ambiguity-level errors. A similar result has been obtained by another investigator [17]. This is because of its ability to work across and around low-coherence regions, which results in more accurate connecting of the high-coherence regions. This results in a higher proportion of the interferogram being unwrapped, and more regions being joined into one contiguous region.

While we believe that the region-growing algorithm is effective on the difficult Sardegna interferogram, it should be stated that this effectiveness comes with a considerable computing time penalty for difficult interferograms.

\section{ACKNOWLEDGMENT}

The authors would like thank H. Lim and L. Keong Kwoh of the Center for Remote Imaging and Sensing, National University of Singapore, for early discussions and encouragement, and M. Seymour and J.-L. Valero of the Radar Remote Sensing Group, University of British Columbia, for helpful discussions. The phase unwrapping results were provided by I. Joughin of the Jet Propulsion Laboratory ("Cut-Line" algorithm) and by M. Schwaebish of the German Aerospace Research Establishment, DLR ("Weighted Least Squares" algorithm). They are grateful to the European Space Agency for supplying the ERS-1 data. The authors would also like to thank D. Stevens of Macdonald Dettwiler and Associates and P. Vachon of Canada Center for Remote Sensing for helpful suggestions on the manuscript.

\section{REFERENCES}

[1] D. W. Robinson and G. T. Reid, Interferogram Analysis-Digital Fringe Pattern Measurement Techniques. $\quad$ Philadelphia, PA: Inst. Phys., 1993.

[2] R. M. Goldstein, H. A. Zebker, and C. L. Werner, "Satellite radar interferometry: Two-dimensional phase unwrapping," Radio Sci., vol. 23, pp. 713-720, July/Aug. 1988.

[3] Q. Lin, J. F. Vesecky, and H. A. Zebker, "New approaches in interferometric SAR data processing," IEEE Trans. Geosci. Remote Sensing, vol. 30, pp. 560-567, May 1992

[4] C. Prati, M. Giani, and N. Leuratti, "A 2-D phase unwrapping technique based on phase and absolute value information," in IGARSS'90, Washington, DC, pp. 2043-2046.

[5] D. G. Ghiglia and L. A. Romero, "Robust two-dimensional weighted and unweighted phase unwrapping that uses fast transforms and iterative methods," J. Opt. Soc. Amer. A., vol. 11, no. 1, pp. 107-117, 1994.

[6] J.-L. Valero and I. Cumming, "A survey of phase unwrapping techniques, with applications to SAR," Dept. Elect. Eng., Univ. British Columbia, Vancouver, B.C., Canada, Tech. Rep., June 1995.

[7] H. Lim, W. Xu, and X. Huang, "Two new practical methods for phase unwrapping," in Proc. IGARSS'95, Firenze, Italy, pp. 196-198.

[8] W. Xu and I. Cumming, "A region growing algorithm for InSAR phase unwrapping," in Proc. IGARSS'96, Lincoln, NE, pp. 2044-2046.

[9] H. A. Zebker, C. L. Werner, P. A. Rosen, and S. Hensley, "Accuracy of topographical maps derived from ERS-1 interferometric radar," IEEE Trans. Geosi. Remote Sensing, vol. 32, pp. 823-836, July 1994.

[10] ERS, ESRIN, Proc. ERS-1 FRINGE, 1st Workshop, SAR Interferometry Working Group, Frascati, Italy, Oct. 1992

[11] A. Sowter and H. D. Groof, "Co-registration and phase unwrapping techniques," in Proc. ERS-1 Fringe, 1st Workshop, SAR Interferometry Working Group Frascati, Italy, Oct. 1992.

[12] D. G. Ghiglia and L. A. Romero, "Minimum Lp-norm two-dimensional phase unwrapping," J. Opt. Soc. Amer. A, vol. 13, no. 1, pp. 1-15, 1996.

[13] M. D. Pritt, "Phase unwrapping by means of multigrid techniques for interferometric SAR," IEEE Trans. Geosci. Remote Sensing, vol. 34, pp. 728-738, May 1996.

[14] G. W. Davidson and R. Bamler, "A multiresolution approach to improve phase unwrapping," in Proc. IGARSS'96, Lincoln, NE, pp. 2050-2053.

[15] T. J. Flynn, "Consistent 2-D phase unwrapping guided by a quality map," in Proc. IGARSS'96, Lincoln, NE, pp. 2057-2059.

[16] M. Costantini, "A phase unwrapping method based on network programming," in Proc. FRINGE'96, ESA Workshop ERS SAR Interferometry, Univ. Zurich, Zurich, Switzerland.

[17] M. D. Pritt, "Comparison of path-following and least-squares phase unwrapping algorithms," in Proc. IGARSS'97, Singapore, pp. 872-874.

[18] G. A. Solaas, "ERS-1 interferometric baseline algorithm verification," Eur. Space Agency, Tech. Rep. ES-TN-DPE-OM-GS02, version 1.0, 1994, available at http://gds.esrin.esa.it:80/CEFB565F/CORBITS.

[19] I. Joughin, "Estimation of ice-sheet topography and motion using interferometric synthetic aperture radar," Ph.D. dissertation, Univ. Washington, Seattle, 1995. 
[20] M. Schwabisch, M. Matschke, W. Knopfle, and A. Roth, "Quality assessment of InSAR-derived DEM's generated with ERS tandem data," in Proc. IGARSS'96, Lincoln, NE, pp. 802-804.

[21] R. Bamler, N. Adam, G. W. Davidson, and D. Just, "Noise-induced slope distortion in 2-D phase unwrapping by linear estimators with application to SAR interferometry," IEEE Trans. Geosci. Remote Sensing, vol. 36, pp. 913-921, May 1998.

[22] I. Cummings and W. Xu, "Comparison of phase unwrapping using a noisy interferogram," in Proc. 3rd ERS Scientific Symp., Florence, Italy, Mar. 17-20, 1997, published electronically at http://florence97.erssymposium.org/.

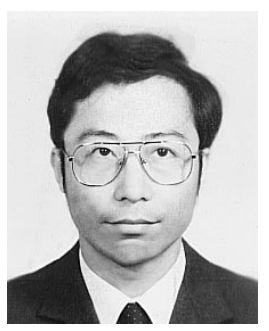

Wei Xu (M'94) received the B.A.Sc. and M.A.Sc. degrees in electrical engineering from the University of Science and Technology of China (USTC) in 1982 and 1984, respectively, and the Ph.D. degree in electrical engineering from the Institute of Electronics, Chinese Academy of Sciences (IECAS), in 1989.

He produced the first digital SAR image in China in 1984, while working toward the M.A.Sc. degree. From 1984 to 1985, he taught in the Department of Radio and Electronics, USTC. From 1988 to 1993, he was with IECAS as an Assistant Research Professor. During this period, he was an Assistant Project Leader, the Group Leaders for three subsystems, and a Group Co-Leader for another subsystem in the project of real-time digital SAR imaging processor development. From 1993 to 1995, he was a Research Fellow at the Center for Remote Imaging, Sensing, and Processing (CRISP), National University of Singapore (NUS), where he worked on SAR and InSAR processing and applications. From 1995 to 1997, he was a Scientist at the Radar Remote Sensing Group (RRSG), University of British Columbia (UBC), Vancouver, B.C., Canada, where he worked on SAR/InSAR processing and simulation. He is currently a Software Engineer at MacDonald, Detwiler and Associates Ltd., Richmond, B.C.

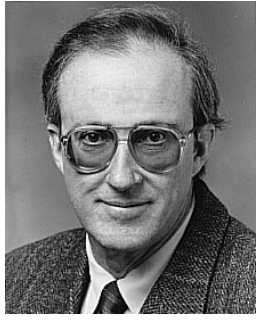

Ian Cumming (S'63-M'66) received the B.Sc. degree in engineering physics at the University of Toronto, Toronto, Ont., Canada, in 1961 and the $\mathrm{Ph} . \mathrm{D}$. degree in computing and automation from Imperial College, University of London, London, U.K., in 1968.

He joined MacDonald Dettwiler and Associates, Richmond, B.C., Canada, in 1977, following work in steel mill automation and sonar signal processing. Since that time, he has developed SAR signal processing algorithms and worked on systems for processing polarimetric and interferometric radar data and for the compression of radar data. He has been involved in the algorithm design of the digital SAR processors delivered by MacDonald Dettwiler for SEASAT, SIR-B, ERS-1/2, J-ERS-1, and Radarsat, as well as several airborne radar systems. In 1993, he joined the Department of Electrical and Computer Engineering, University of British Columbia, Vancouver, where he holds the MacDonald Dettwiler/NSERC Industrial Research Chair in radar remote sensing. The Radar Remote Sensing Laboratory supports a research staff of eight engineers and students, working in the fields of SAR processing, SAR data encoding, satellite SAR two-pass interferometry, airborne along-track interferometry, airborne polarimetric radar classification, and SAR Doppler estimation. He and his associates are principal investigators on the ERS Tandem Mission and the Radarsat ADRO. 